\title{
The Production of Tyrothricin by Submerged Culture of Bacillus brevis in Synthetic Media
}

\author{
By J. C. APPLEBY, E. KNOWLES, R. C. A. MCALLISTER, \\ J. PEARSON AND T. WHITE \\ The Research Laboratories, John Wyeth and Bro. Ltd., London
}

\begin{abstract}
SUMMARY: Synthetic media containing ammonium succinate as the sole source of nitrogen permit Bacillus brevis to grow and form tyrothricin in aerated submerged culture. The yield of the antibiotic is almost doubled by adding a complex of B-vitamins to the medium, the effect being due specifically to the presence of biotin.

Urea can be used as an alternative but less satisfactory source of nitrogen, but ammonium succinate cannot be replaced by ammonium salts of a number of other organic acids. The process can be operated successfully on a large scale.

Normally, B. brevis will grow, but will not produce tyrothricin, in submerged culture in the presence of complex nitrogen sources. An exception to this has been observed, but metabolic investigations show distinct differences in the behaviour of the organism (in submerged culture) in synthetic and in peptone media.
\end{abstract}

An outline of previous work on the submerged-culture formation of tyrothricin has been given in the preceding paper (Appleby, Knowles, Pearson \& White, 1947). It was then decided to aim at developing a synthetic medium containing ingredients which would provide Bacillus brevis with nitrogen sources capable of supporting the production of tyrothricin in submerged culture, and which would be available in such quantity and at such cost as would permit of the use of the method on a large scale. This aim was realized.

\section{EXPERIMENTAL}

\section{Methods}

In all experiments $6 \mathrm{l}$. of medium was made up in a $10 \mathrm{l}$. aspirator, the lower outlet of which was fitted with a rubber stopper carrying a thermometer, a sampling tube with external cotton-wool cover, and a glass aeration tube extending horizontally into the aspirator about 1 in. above the bottom and terminating in a number of fine jets. This tube was connected to a compressed air line via a cotton-wool packed section for air filtration, and a 'Rotameter' for measuring the air flow. The aspirator outlet was closed by a rubber stopper carrying a short vertical $\frac{1}{2}$ in. bore glass tube covered with a wired-on external cotton-wool plug. This tube served for adding the inoculum, for periodic addition of anti-foam (sterilized $2 \%(w / v)$ stearyl alcohol in lard oil), and as an air outlet. The medium was adjusted to the required $\mathrm{pH}$ (usually 6.5 ) before sterilizing, and the whole assembly steam-sterilized at $12 \mathrm{lb}$./sq.in. pressure for $30 \mathrm{~min}$. After cooling, the $\mathrm{pH}$ and the medium constituents were checked and the aspirator inoculated in a 'sterile area' (Grenfell, Legge \& White, 1947). Each aspirator was inoculated with $10 \mathrm{ml}$. from a 3-day peptone-yeast extract broth culture of $B$. brevis, and was then placed in an incubation room at $37^{\circ}$ and connected to the compressed air line. Air was supplied at $20 \mathrm{lb}$. pressure and controlled at a rate 
of $1 \mathrm{l}$. free air/l. medium/min., the resultant agitation making stirring unnecessary. Samples were taken at intervals by removing the external cotton-wool plug of the sampling tube, flaming the end of the latter, and then allowing the requisite volume of culture fluid to run out into a sterile sampling flask, the first $20 \mathrm{ml}$. sample being discarded. Analyses were carried out for $\mathrm{pH}$, glucose, ammonia, turbidity and tyrothricin content. The last named was determined by the assay method of Appleby et al. (1947), by direct isolation of tyrothricin, or by both methods. The $\mathrm{pH}$ was determined electrometrically, turbidity with a 'Spekker' photoelectric absorptiometer $(1 \mathrm{~cm}$. cell), ammonia by micro-Kjeldahl, and glucose by the method of Schaffer \& Hartman (1920). Turbidity, glucose and pH determinations were carried out directly on portions of the sample; ammonia determinations were made with samples of clear supernatant after centrifuging off the bacterial cells. Tyrothricin isolations were made by the method described by Appleby et al. (1947).

\section{Preliminary experiments}

Submerged-culture experiments were carried out with our strain $\mathrm{F}$ of the Dubos B.G. strain of $\boldsymbol{B}$. brevis using initially the following media: $(a)$ that used previously for surface culture-i.e. peptone $10 \mathrm{~g}$., yeast extract $3 \mathrm{~g}$., $\mathrm{NaCl} 5 \mathrm{~g}$., water to 1 l., pH 7.0; (b) that of Stokes \& Woodward (1943) with glutamic acid as the nitrogen source-i.e. glutamic acid 5 g., glucose 10 g., inorganic salts (see below), water to $1 \mathrm{l}, \mathrm{pH} 6.4 ;(c)$ as $(b)$ with glutamic acid substituted by asparagine $(2.5 \mathrm{~g} . / \mathrm{l}$.$) ; (d)$ as $(b)$ with the addition of Difco yeast extract (3 g./l.). In conformity with the results of Stokes \& Woodward (1943) good growth occurred, but no tyrothricin was formed in medium (a). At a later date, however, our strain $\mathrm{F}$ did develop the capacity of producing tyrothricin in peptone media in submerged culture, and this point will be discussed later. The glutamic acid medium $(b)$ gave a tyrothricin yield of $0.30 \mathrm{~g}$./l. culture, while the asparagine medium $(c)$ gave $0.21 \mathrm{~g}$./1. In medium $(d)$ growth of the organism was obviously much greater than in medium $(b)$, but the presence of the yeast extract completely inhibited the formation of tyrothricin.

\section{. Ammonium salts of organic acids as sources of nitrogen}

Attempts were next made to produce tyrothricin by aerated submerged growth of $B$. brevis in media containing ammonium salts of organic acids as the only sources of nitrogen, glucose being used as the primary carbon source with the usual inorganic salts also present. Attention was focused on ammonium succinate, citrate, tartrate, lactate and carbamate, each being used separately at a level of $5 \mathrm{~g}$./l. in a medium containing also (g./l.): glucose $10 ; \mathrm{K}_{2} \mathrm{HPO}_{4} \mathbf{0 . 5}$; $\mathrm{KH}_{2} \mathrm{PO}_{4} 0 \cdot 5 ; \mathrm{MgSO}_{4}, 7 \mathrm{H}_{2} \mathrm{O} 0 \cdot 2 ; \mathrm{NaCl} 0.01 ; \mathrm{FeSO}_{4}, 7 \mathrm{H}_{2} \mathrm{O} 0 \cdot 01 ; \mathrm{CaCl}_{2} \mathbf{0} 25$.

Each aspirator was run at $37^{\circ}$ with equal volume aeration for at least $150 \mathrm{hr}$. The ammonium lactate and carbamate media failed to support growth of the organism. Sparse growth occurred in the ammonium tartrate medium, but no tyrothricin could be isolated nor could any antibacterial activity be demonstrated in the culture fluid. Both the ammonium citrate and succinate media permitted good growth, and the analytical data showed appropriate utilization of nutrients. With the former salt no antibacterial activity developed nor could any tyrothricin be isolated. The ammonium succinate medium, on the other 
hand, showed a progressively increasing antibacterial effect and gave a final yield of tyrothricin of $0.5 \mathrm{~g}$./l. culture. Repetitions of these experiments have consistently given identical results except that occasionally the tyrothricin yield from the ammonium succinate medium fell to $\mathbf{0 \cdot 3} \mathrm{g} . / \mathrm{l}$.

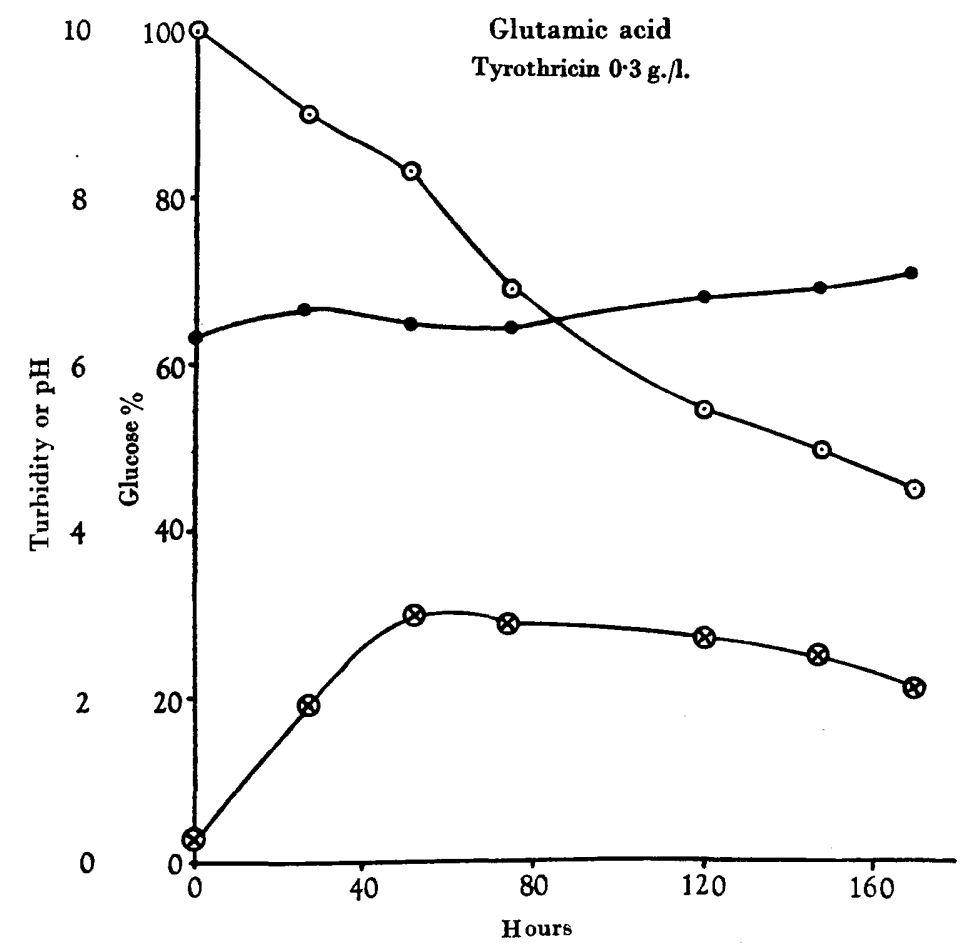

Fig. 1

Figs. 1-3. Metabolism of $B$. brevis in synthetic media with the stated nitrogen sources. Glucose utilization $(\%),-\odot-\bigcirc-$; ammonia utilization $(\%), \ldots \odot \ldots . . \odot \ldots$ pH, $-\bigcirc-$ - ; turbidity (Spekker units-1 cm. cell), $-\otimes-\bigotimes-$; tyrothricin content (g./1.), ........ Final tyrothricin yield as stated.

It is not clear why, of the range of salts tested, ammonium succinate alone should be specific for tyrothricin production, nor why it should permit of higher yields than are obtained with amino-acids as nitrogen sources. In Figs. 1-3 the course of the fermentations in glutamic acid, ammonium citrate, and ammonium succinate media is shown diagrammatically. Ammonia values are not given for glutamic acid since the free ammonia content remained remarkably low and constant throughout the fermentation, indicating either an absence of deamination or immediate and complete utilization of the ammonia liberated by deamination.

The behaviour of the organism in the glutamic acid medium conforms with that outlined by Stokes \& Woodward (1943). The ammonium citrate and ammonium succinate media give virtually identical metabolic pictures except that tyrothricin was produced only in the succinate medium. In both cases 


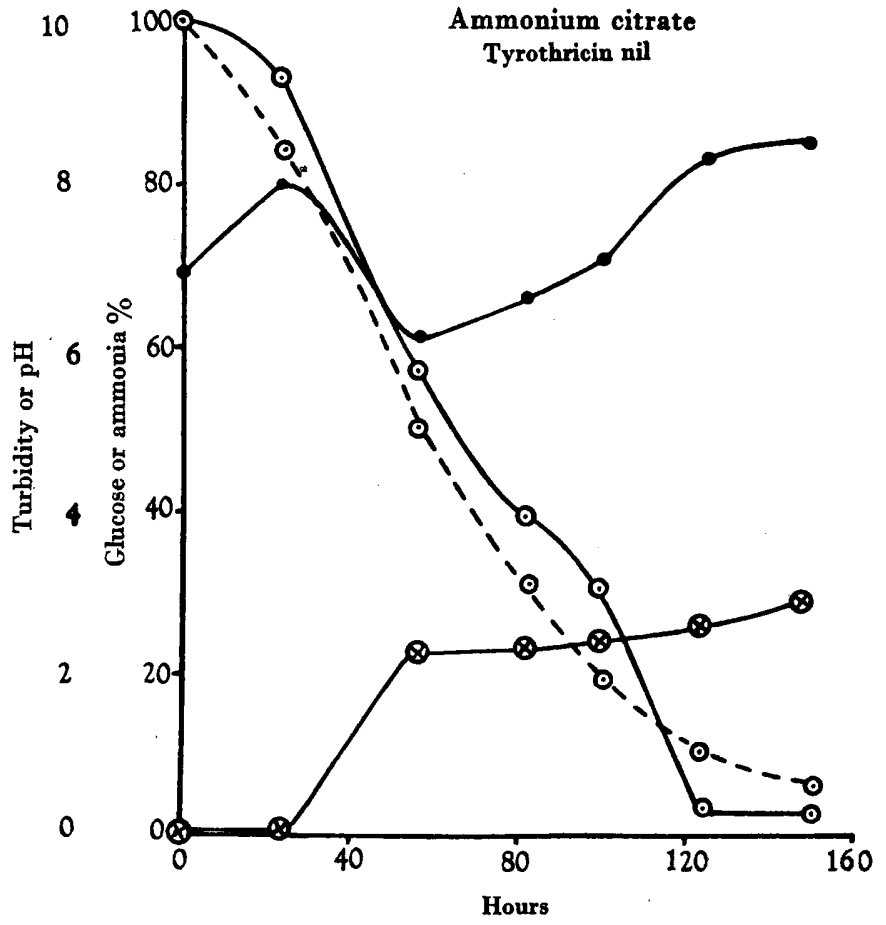

Fig. 2

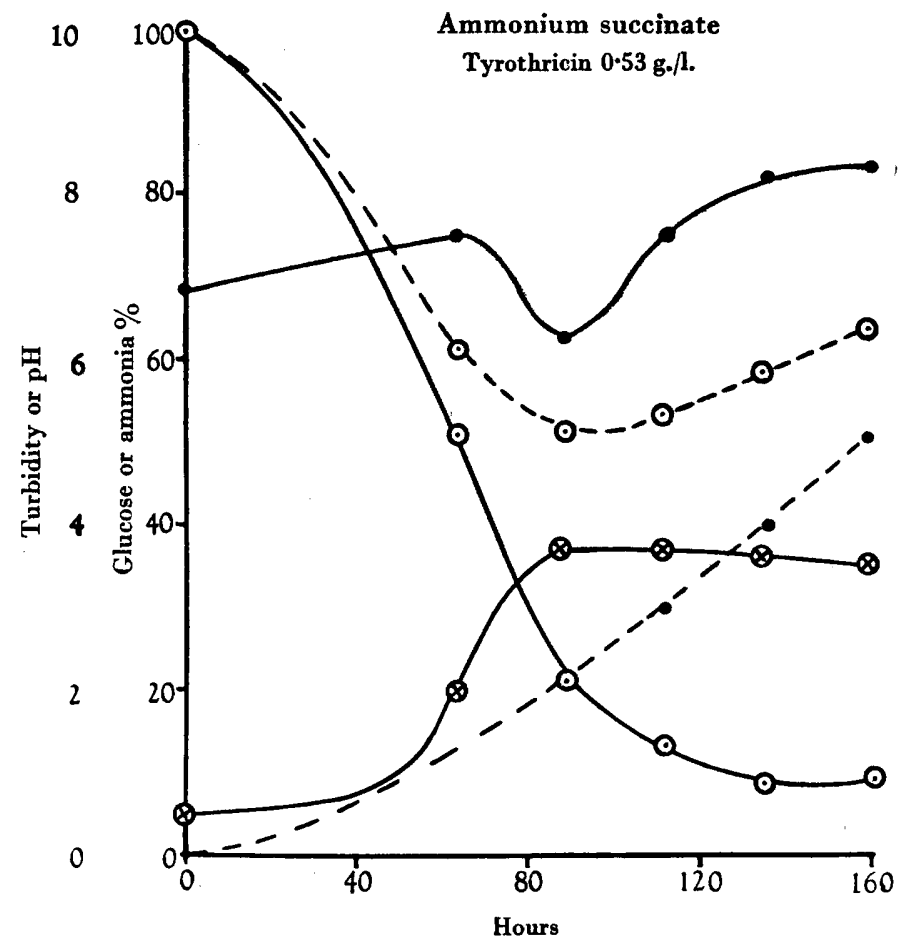

Fig. 3 
an initial rise of $\mathrm{pH}$ was followed by a marked drop with a final rise usually to at least $\mathrm{pH}$ 8.5. Duplicate experiments rarely give identical curves, the degree of rise and fall of $\mathrm{pH}$ and the time at which the changes occur varying somewhat; but the general form of the changes was always as described. In the ammonium succinate medium variation of the initial $\mathrm{pH}$ between 5.8 and 7.5 had no effect on the final yield of tyrothricin, on the rate of use of nutrients, or on the rate of growth of the organism. The only feature calling for comment is that the higher the initial $\mathrm{pH}$, the less marked was the first rise, and this perhaps would be expected.

The glucose utilization curves generally show a brief initial lag which, owing to loss of water by evaporation, may show up as an initial increase of the sugar concentration. Thereafter, utilization is rapid and almost complete by $120 \mathrm{hr}$., but the curve then flattens with some 5-10\% of the initial sugar remaining unused. The phase of rapid glucose utilization is generally coincident with the fall of $\mathrm{pH}$ and, from several experiments with ammonium succinate, it appears that this $\mathrm{pH}$ fall is more extreme in runs showing the most rapid glucose metabolism. The final $\mathrm{pH}$ rise generally begins when carbohydrate decomposition is approaching completion, suggesting that acid accumulates during the active growth phase.

Ammonia utilization, in the case of the ammonium citrate medium, appears to follow that of glucose very closely. In the ammonium succinate medium, however, two distinct types of ammonia metabolism occur: (i) ammonia disappearance parallels that of glucose throughout; (ii) after about half the ammonia has been used ( $80 \mathrm{hr}$.), the ammonia concentration rises again (Fig. 3): this type is the more frequent. Such differences are found even in simultaneous replicate experiments, and do not appear to be correlated with special changes of $\mathrm{pH}$, glucose metabolism or growth rate; the yield of tyrothricin is not affected. It was noted that, in most experiments, type (i) was accompanied by poor sporing, while in type (ii) sporing began early and became almost complete. However, this was not always the case, so it is not possible to conclude that the secondary formation of ammonia is the result of autolysis.

The turbidity data for both citrate and succinate media show an initial lag phase lasting for some $40 \mathrm{hr}$., followed by a similar period of rapid growth; after $80 \mathrm{hr}$. turbidity remains unchanged or falls slightly. In general, maximum turbidity coincides with the beginning of sporulation and occurs well before the various nutrients are exhausted.

The rate of tyrothricin formation in ammonium succinate media is also shown in Fig. 3. The final yield is not improved by further incubation.

\section{Effect of addition of vitamin B-complex}

It was shown by Appleby et al. (1947) that the addition of yeast extract to peptone medium markedly increased the yield of tyrothricin obtained in surface culture. In submerged culture (glutamic acid medium) the growth of B. brevis is stimulated by yeast extract, but the formation of tyrothricin is inhibited 
(see p. 146). Since it seems probable that the stimulation of growth is due to the presence of B-vitamins in the extract, whereas the inhibition of tyrothricin formation is due to the presence of proteins or their degradation products, it was decided to test the result of adding a product containing the members of the vitamin B-complex but free from proteins, amino-acids, etc. The commercial product 'B-Plex' (John Wyeth and Bro. Ltd., 25 Oldhill Place, London. N. 16)

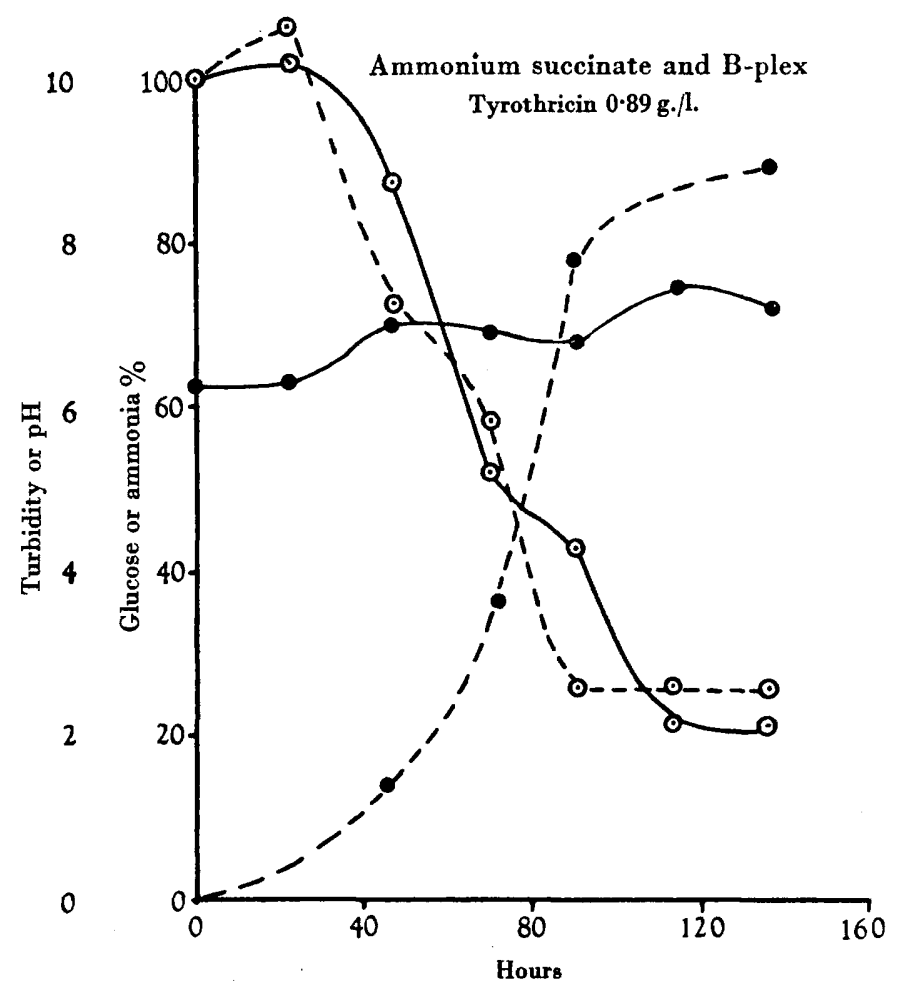

Fig. 4. Metabolism of $B$. brevis in ammonium succinate medium plus $0.5 \%$ B-Plex. Symbols as for Figs. 1-3.

met these requirements. It is a fortified extract of rice bran containing ( $\mu \mathrm{g} . / \mathrm{ml}$.$) : aneurin hydrochloride 125$, riboflavin 250, nicotinic acid 1250 , pyridoxin hydrochloride 125 , calcium pantothenate 625 , choline 3000 , inositol 1800 , and biotin 1 .

The addition of $0.5 \%(\mathrm{v} / \mathrm{v})$ of this product to the ammonium succinate medium stimulated growth of $\boldsymbol{B}$. brevis, and also markedly increased the yield of tyrothricin, $c .0 .9 \mathrm{~g}$./l. culture medium being obtained. Comparison of Figs. 3 and 4 shows that, although the rate of utilization of nutrients is not markedly affected, both the rate of formation and the final yield of antibiotic are increased. It appears therefore that, in submerged (though not in surface) culture, the effect of B-vitamins in increasing tyrothricin production is only apparent if proteins and amino-acid mixtures are absent.

Pilot-plant scale runs with the succinate-B-Plex medium were carried out 
in a glass-lined $200 \mathrm{l}$. fermentation vessel designed for larger scale work on antibiotics (Grordon, Grenfell, Knowles, Legge, McAllister \& White, 1947). In general, similar results were obtained except that the yields of tyrothricin were lower $(0 \cdot 3-0 \cdot 4 \mathrm{~g} . / 1$.$) , but were obtained in a shorter period (120 hr.). The$ general form of the results makes it clear that the findings in aspirators can be applied, with proper technical modifications, to larger scale working.

The question as to which of the vitamins causes these effects was next investigated in aspirator-scale experiments. The inoculum for these tests was $10 \mathrm{ml}$. from a 3-day surface culture on the basal ammonium succinate medium. In each fermentation a single vitamin was added in the amount given below, up to six experiments being conducted simultaneously in order to obtain results as comparable as possible; a control experiment without added vitamin was included in each set. A typical series of results is given in Table 1; the vitamin concentrations used were equivalent to those present in the experiments with B-Plex.

Table 1. Effect of vitamins of the B-complex on tyrothricin production

Basal medium (g./1.): ammonium succinate $5 \cdot 0$; glucose $10 \cdot 0 ; \mathrm{KH}_{2} \mathrm{PO}_{4} 0.5 ; \mathrm{K}_{2} \mathrm{HPO}_{4} 0.5$; $\mathrm{MgSO}_{4}, 7 \mathrm{H}_{2} \mathrm{O} 0.2 ; \mathrm{NaCl} 0.01 ; \mathrm{FeSO}_{4}, 7 \mathrm{H}_{2} \mathrm{O} 0.01 ; \mathrm{CaCl}_{2} 0.25$.

Yield of

Vitamin added

Amount tyrothricin

Aneurin hydrochloride (mg./l. medium) (g./l. medium)

Nicotinic acid

$0 \cdot 625$

$6 \cdot 250$

$0 \cdot 60$

Pyridoxin

0.625

0.55

Pantothenic acid

$3 \cdot 125$

$0 \cdot 60$

Choline hydrochloride

$15 \cdot 000$

$0 \cdot 34$

Riboflavin

$\mathbf{1} \cdot \mathbf{2 5 0}$

0.36

Inositol

$9 \cdot 000$

$0 \cdot 43$

Biotin

0.005

$0 \cdot 29$

$0 \cdot 88$

None

0.53

The analytical data obtained with pyridoxin and biotin are illustrated in Figs, 5 and $\mathbf{6}$, and may be compared with those for unsupplemented ammonium succinate (Fig. 3). In general, for all the separate vitamin runs, the data conformed closely to those for ammonium succinate without added vitamins. Ammonia utilization is rapid and almost complete in the case of pantothenic acid, choline or inositol; but is of the incomplete type in the case of the other vitamins. As indicated previously, however, the occurrence of one or the other type of ammonia metabolism is of uncertain significance. Figs. 5 and 6 have been chosen because they illustrate a feature of particular interest. The addition of biotin, riboflavin or inositol (in the amounts stated in Table 1) consistently delays the initial stages of the fermentation, and little utilization of glucose or ammonia takes place for $c .80 \mathrm{hr}$. (Fig. 6). Growth, as indicated by turbidity, shows a similar lag. Thereafter, the rate of metabolism is faster than usual, and by $120 \mathrm{hr}$. these fermentations have reached the same stage as the controls or those with other single vitamins present.

Pyridoxin, aneurin, nicotinic acid, pantothenic acid and choline have little, if any, effect on the rate of metabolism or growth of the organism (e.g. pyri- 


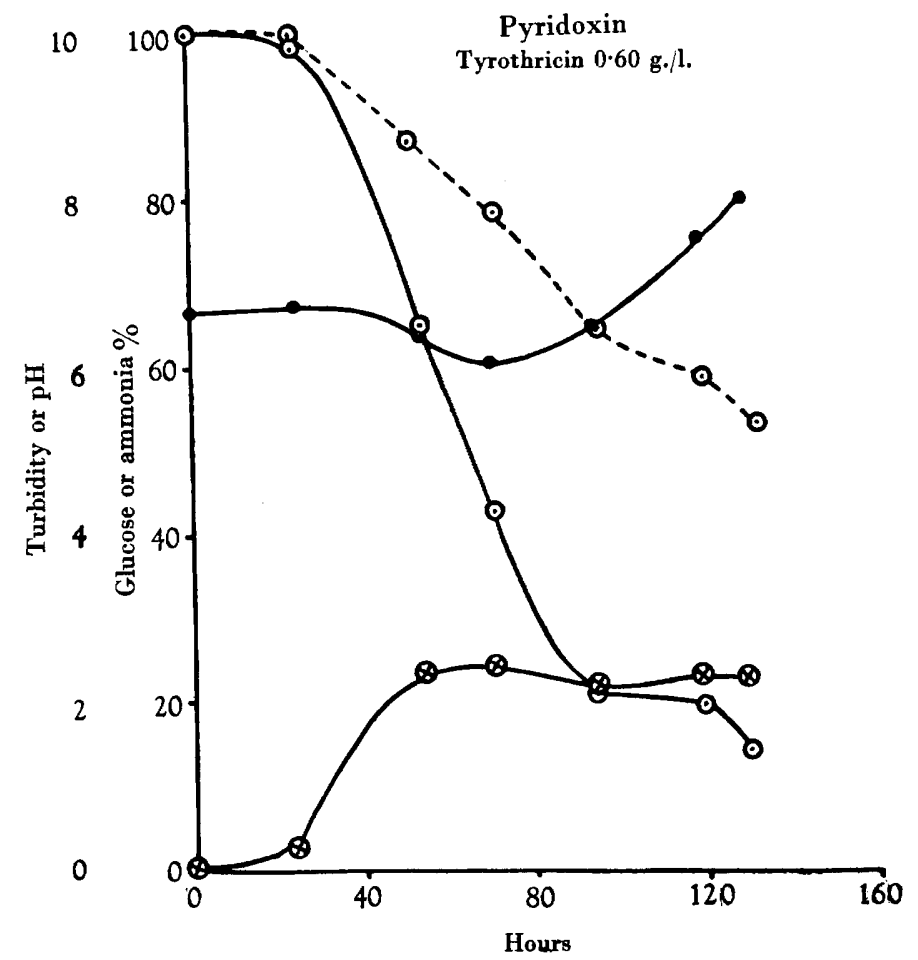

Fig. 5

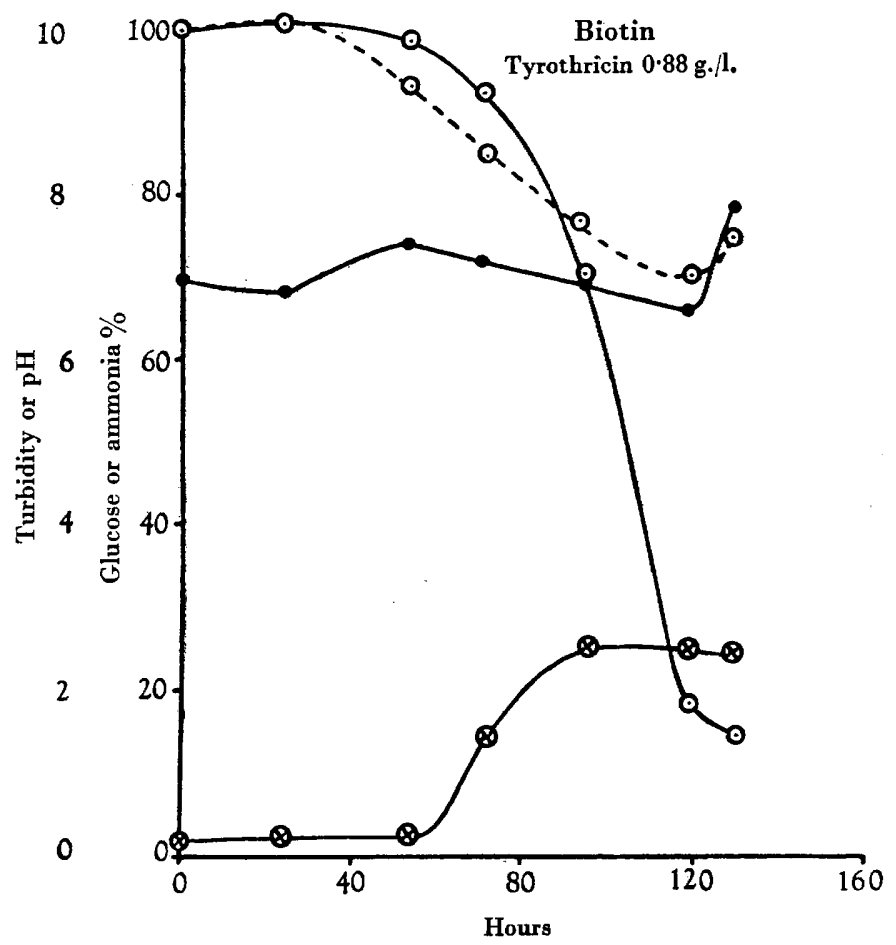

Fig. 6

Figs. 5, 6. Metabolism of $B$. brevis in ammonium succinate medium in the presence of pyridoxin and biotin. Symbols as for Figs. 1-3. 
doxin, Fig. 5). It would seem, therefore, that the stimulating effect of BPlex on the growth of $\boldsymbol{B}$. brevis in the ammonium succinate medium is a composite one, and is not due to any single member of the vitamin B group. When all the vitamins are present, the initial delaying effect of biotin, riboflavin and inositol is not evident.

It is, however, clear from Table 1 and Fig. 6 that the stimulating effect of B-Plex on the tyrothricin yield is due to the presence of biotin, since addition of the latter alone $(5 \mu \mathrm{g}$. $/ \mathrm{l}$.) brings the yield of tyrothricin up to $0.88 \mathrm{~g}$./l. medium as compared with the $0.89 \mathrm{~g}$. $/ 1$. obtained with B-Plex. There is evidence that aneurin and pyridoxin may aid in increasing the tyrothricin yield, as each give $0.60 \mathrm{~g}$. $/ 1$. as against controls of $0.53 \mathrm{~g}$./l. The yield with nicotinic acid is unchanged, while riboflavin, pantothenic acid, choline and inositol all appear to decrease slightly the ultimate yield of tyrothricin or, alternatively, may merely delay its formation. It is clear from this that the stimulation or otherwise of the yield of tyrothricin cannot be predicted from the utilization of nutrients, and must be due to some more subtle effect on metabolism. Observation of the morphological appearance of the organism throughout each run cast no light on this point.

It is not proposed here to discuss further why biotin should have such a marked stimulating effect on the yield of tyrothricin, nor to relate this observation to previous work on the effect of vitamins on micro-organisms. The latter field has been fully reviewed recently by Peterson \& Peterson (1945), but the only work with $B$. brevis appears to be that of Landy, Dicken, Bicking \& Mitchell (1942), who found that biotin had a marked effect on metabolism. In view of the potential importance of this organism and the definite link between the action of biotin and the formation of tyrothricin, more detailed investigation of this relationship is desirable.

\section{Urea as source of nitrogen}

Investigations were also carried out with a synthetic medium in which the ammonium succinate was replaced by a corresponding amount of urea as the nitrogen source. The organism would not always grow satisfactorily on this medium, but when growth did occur it was accompanied by tyrothricin formation, the yields being $c .0 \cdot 2 \mathrm{~g}$./1. Fig. 7 shows that the metabolic picture again has its distinctive features. There is a lag phase of some $80 \mathrm{hr}$. before glucose and ammonia utilization commence properly, and little change of $\mathrm{pH}$ or turbidity occurs during this period. Thereafter utilization is rapid but falls off at higher concentrations than in the succinate medium, possibly because in the urea experiments the $\mathrm{pH}$ tends to fall to levels unfavourable to optimum growth and does not show the final steep rise which occurs in a really suitable medium.

\section{Tyrothricin formation in peptone medium}

After our strain F of B. brevis B.G. (Dubos) had been cultured for some 18 months, it suddenly developed the capacity to produce tyrothricin in deep culture in peptone media. This finding was unexpected, and resulted from a 
decision to investigate more closely the metabolism of the organism in the presence of peptone. Fig. 8 gives the analytical data for an experiment with the peptone-yeast extract medium described earlier. This experiment gave a tyrothricin yield of $0.20 \mathrm{~g} . / 1$. medium. The results of a similar experiment in the same medium with the addition of glucose $(10 \mathrm{~g} . / 1$.$) are given in Fig. 9;$ the tyrothricin yield was $0 \cdot 15 \mathrm{~g} . / \mathrm{l}$.

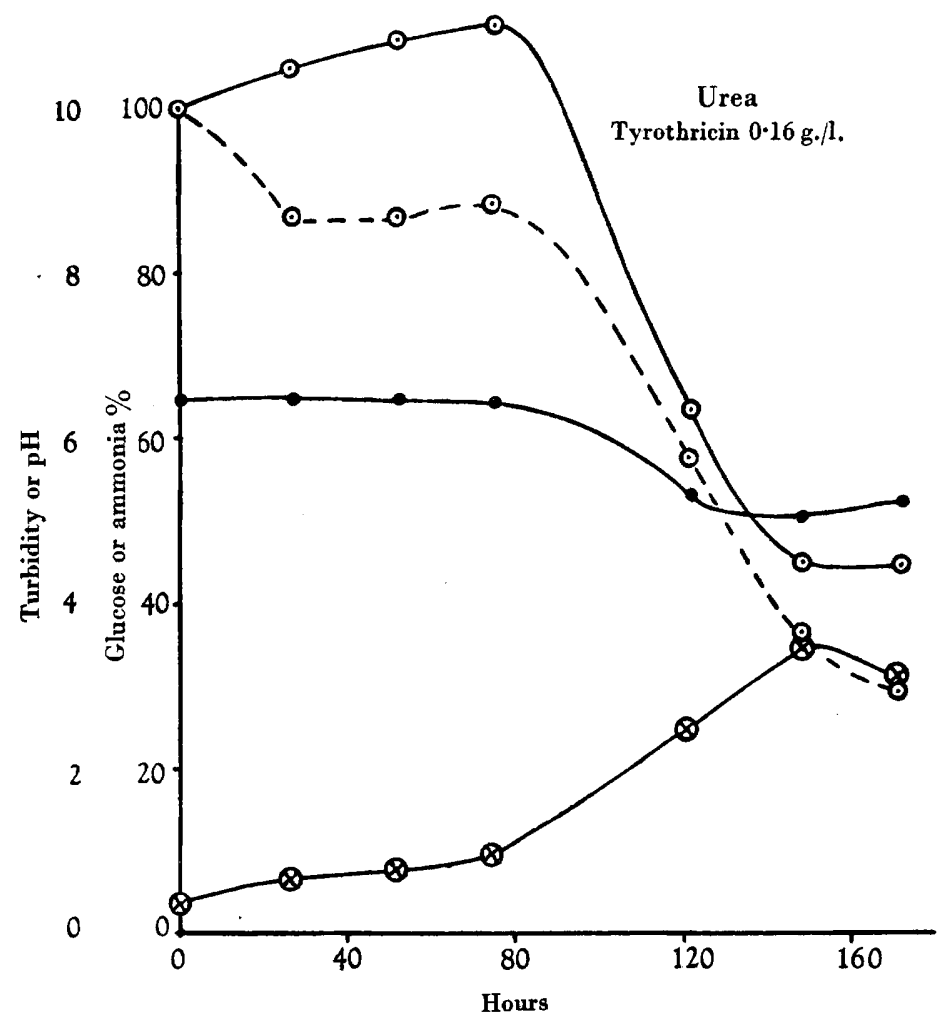

Fig. 7

Figs. 7-9. Metabolism of $B$. brevis in the presence of urea, peptone, and peptone + glucose, respectively. Symbols as for Figs. 1-3, except in Figs. 8 and 9 where ammonia is shown as $\mathrm{mg} . / 100 \mathrm{ml}$. instead of as percentage utilization.

Both in the presence and absence of glucose there is an initial liberation of ammonia into the medium, presumably as a result of proteolysis. In the medium without glucose this is particularly marked, and reaches a peak by $80 \mathrm{hr}$. Thereafter ammonia disappears at a correspondingly rapid pace, suggesting that the organism does not use ammonia initially in the presence of complex nitrogen sources, but does so later in the fermentation. This may be responsible for the low yield of tyrothricin obtained. To some extent the fall after $80 \mathrm{hr}$. may be due to loss of ammonia by aeration at a marked alkaline $\mathrm{pH}$, but it seems improbable that this could account for the very rapid elimination 
shown in Fig. 8. The pH curve clearly reflects the initial accumulation and subsequent utilization of ammonia and is quite different from the $\mathrm{pH}$ curves for the synthetic media.

In the presence of glucose, the initial accumulation of ammonia is less marked and more prolonged. The fact that glucose is being metabolized is

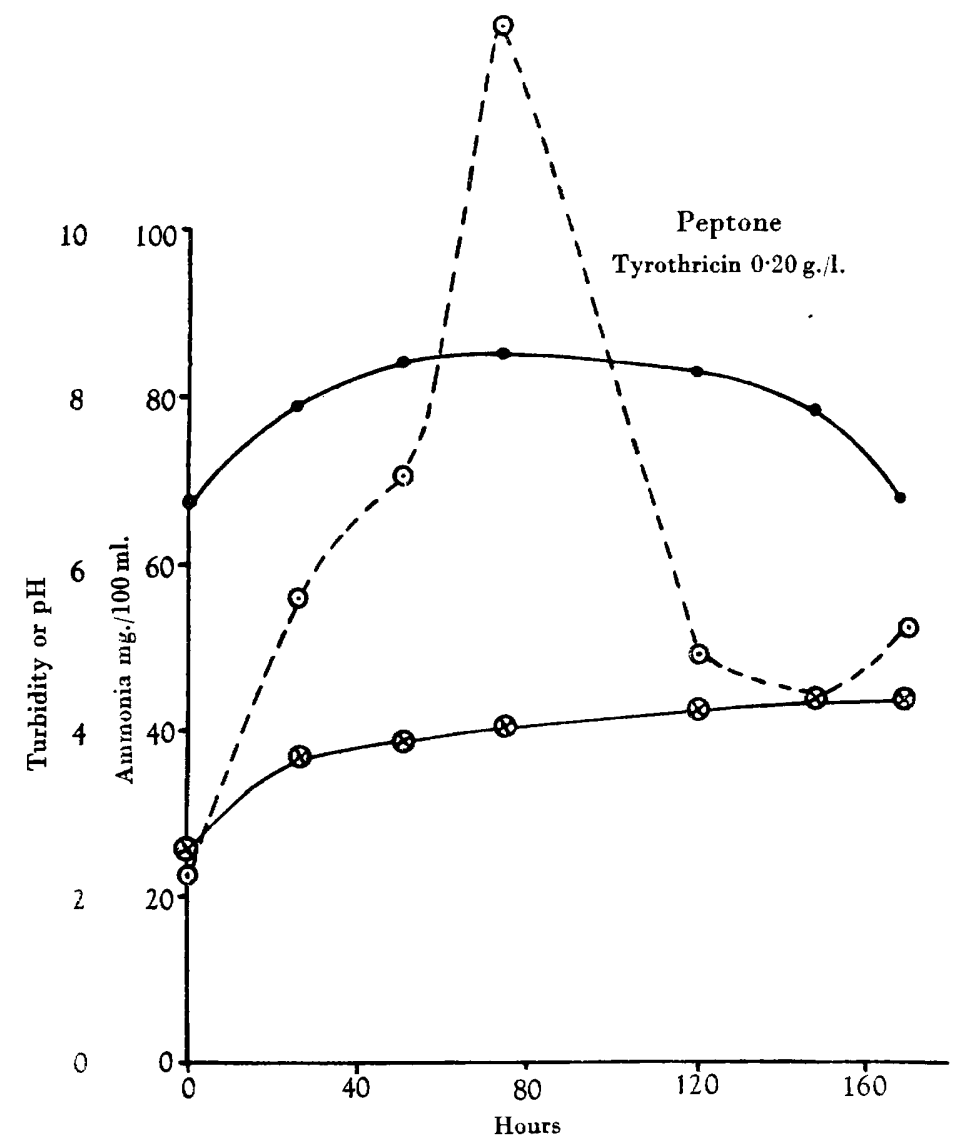

Fig. 8

evidently responsible for this, since the organism is clearly using the sugar as a carbon source in preference to, or in addition to, the peptone. The extent of deamination is presumably less under these conditions, and the peak liberation of ammonia is correspondingly lower. Utilization of ammonia is again not marked until the later stages of the fermentation. The $\mathrm{pH}$ changes are less easy to interpret but seem to indicate an initial production of acid which outweighs ammonia liberation, followed by a rise of $\mathrm{pH}$ which may be due to metabolism of the acidic carbohydrate breakdown products once the initial attack on the glucose molecule is complete. These results support the view of Appleby et al. (1947) that B. brevis cannot be regarded as an organism incapable 
of producing acid from carbohydrates. In all these experiments, the turbidity data do not indicate the initial growth lag found with synthetic media; the initial rate of growth is rapid and slows down to a steady value after $c .50 \mathrm{hr}$.

Despite the differences caused by the presence or absence of glucose, it is clear that in the presence of peptone the metabolism of submerged cultures differs radically from that in synthetic media. In fact, the data suggest that when peptone is present the primary consideration of the organism is its source

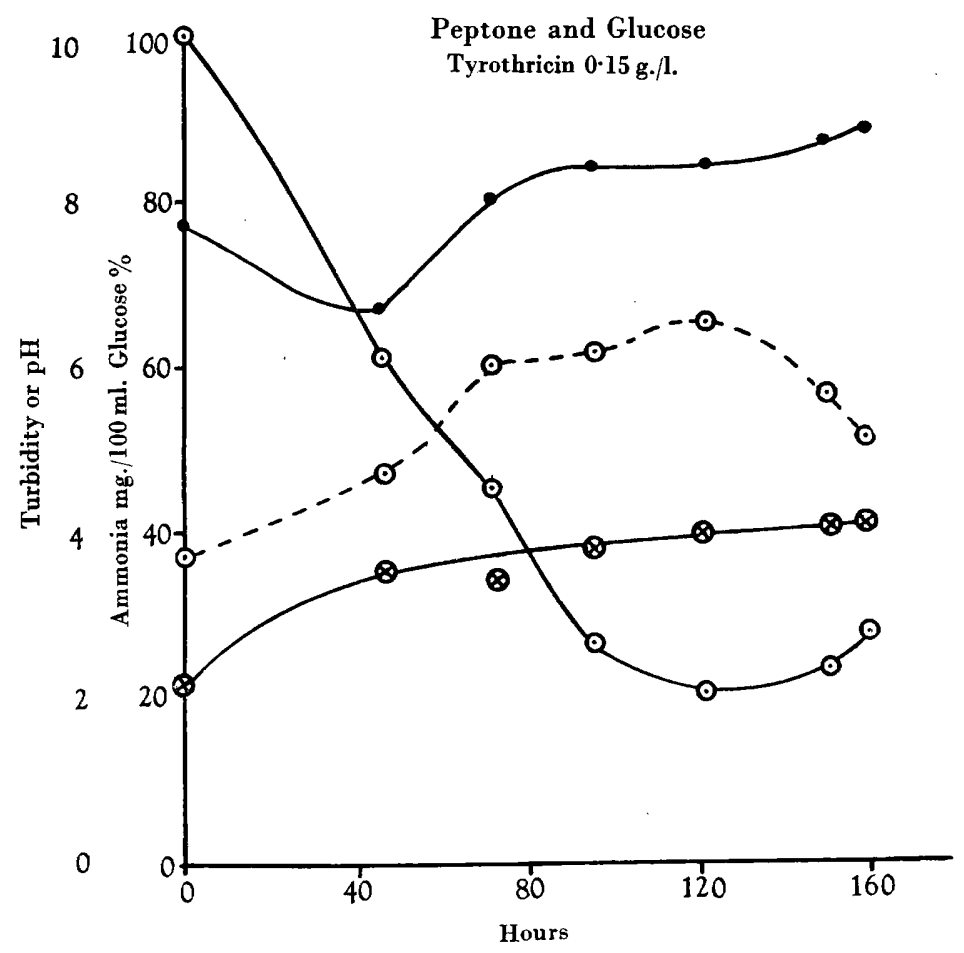

Fig. 9

of carbon rather than of nitrogen. In the synthetic media both appear to call forth an equal degree of metabolic activity, and this may constitute a clue to the fact that normally $\boldsymbol{B}$. brevis grows well but does not form tyrothricin (or does so only to a minor degree) when peptone is present. Even in the above experiments the yield of tyrothricin is only one-quarter of that obtainable in surface culture on the same media in half the time. It is not proposed to discuss the reasons why $\boldsymbol{B}$. brevis should suddenly develop the capacity of producing tyrothricin in submerged culture in the presence of peptone after earlier experiments had failed to demonstrate any such ability. Lewis, Dimick \& Feustel (1945) have also reported briefly the formation of tyrothricin in the presence of peptone in the case of a particular substock of the Dubos B.G. strain which had been maintained as a pure culture for some time. 
The authors wish to express their gratitude to Mr A. J. C. Gormley, Managing Director of John Wyeth and Bro. Ltd., for permission to publish the above results.

\section{REFERENCES}

Appleby, J. C., Knowles, E., Pearson, J, \& Write, T. (1947). A preliminary study of the formation, assay and stability of tyrothricin. J. gen. Microbiol. $1,137$.

Gordon, J. J., Grenfell, E., Knowles, E., Legge, B. J., McAllister, R. C. A. $\&$ White, T. (1947). Methods of penicillin production in submerged culture on pilot-plant scale. J. gen. Microbiol. 1, 187.

Grenfell, E., Legge, B. J. \& White, 'T. (1947). Microbiological aspects of the submerged culture production of penicillin on a pilot-plant scale. J. gen. Microbiol. 1, 171.

Landy, M., Dicken, D. M., Bicking, M. M. \& Mitchell, W. R. (1942). Use of avidin in studies on biotin requirements of micro-organisms. Proc. Soc. exp. Biol., N.Y., 49,441 .

Lewis, J. C., Dimick, K. P. \& Feustel, I. C. (1945). Production of tyrothricin in cultures of Bacillus brevis. Industr. Engng Chem. 37, 996.

Peterson, W. H. \& Peterson, M. S. (1945). Relation of bacteria to vitamins and other growth factors. Bact. Rev. 9, 49.

Schaffer, P. A. \& Hartman, A. F. (1920). The iodimetric determination of copper and its use in sugar analysis. II. Methods for the determination of reducing sugars in blood, urine, milk and other solutions. J. biol. Chem. 45, 365 .

Stokes, J. L. \& Woodward, C. R. (1943). The formation of tyrothricin in submerged cultures of Bacillus brevis. J. Bact. 46, 83.

(i ceived 10 October 1946) 